\title{
Diagnóstico polínico da geoprópolis de Melipona scutel- laris L. (Meliponini, Apidae, Hymenoptera) coletada em uma área de Mata Atlântica no nordeste do Brasil
}

\author{
Pollen diagnosis of the Melipona scutellaris L. (Meliponini, Apidae, Hymenoptera) geopropolis
} collected in an area of Atlantic Forest in Northeast Brazil

Vanessa R. Matos $1^{\oplus \ltimes}$ \& Francisco de Assis R. dos Santos $2^{\oplus}$

\begin{abstract}
1. Faculdade de Geociência, Universidade Federal do Mato Grosso, Cuiabá, Mato Grosso, Brasil

2. Departamento de Ciências Biológicas, Universidade Estadual de Feira de Santana, Feira de Santana, Bahia, Brasil
\end{abstract}

\section{Palavras-chave}

Abelhas nativas. Melissopalinologia.

Produtos das abelhas. Plantas resinosas.

\section{Keywords}

Native bees. Melissopalynology. Bee pro-

ducts. Resinous plants.

\section{Doi}

doi.org/10.33447/paubrasilia.v2i1.19

Recebido em: 08/05/2019

Aceite em: 27/05/2019

\begin{abstract}
Resumo
Melipona scutellaris (uruçu) é endêmica da região nordeste do Brasil, poliniza um número diversificado de espécies de plantas e é importante na manutenção da biodiversidade dos biomas da região. O presente trabalho analisou 16 amostras de geoprópolis produzidas por esta abelha em uma área de Mata Atlântica no município de Entre Rios (Bahia), as quais foram processadas seguindo a técnica de acetólise com modificações sugeridas para geoprópolis. Setenta e cinco (75) tipos polínicos foram encontrados, dos quais 59 foram identificados como pertencentes a 28 famílias botânicas. A família Fabaceae foi a mais importante com 12 tipos polínicos identificados. Os gêneros Cecropia (Urticaceae), Eucalptus (Myrtaceae), Mimosa pudica (Fabaceae) e Myrcia I (Myrtaceae) estiveram presentes em todas as amostras analisadas. Os tipos polínicos Protium heptaphyllum (Buseraceae) e Schinus terebinthifolia (Anacardiaceae) foram ambos utilizados como fonte de resina pelas abelhas, apresentando frequências de distribuição de $56,25 \%$ e $81,25 \%$, respectivamente.
\end{abstract}

\begin{abstract}
Melipona scutellaris (urucu) is endemic to the Northeast region of Brazil, pollinates a diverse number of plant species, and is important in maintaining the biodiversity of the biomes in the area, such as the Atlantic Rainforest. The present work analyzed 16 samples of geopropolis produced by this bee in an area of Atlantic Rainforest in the municipality of Entre Rios (Babia state). For the pollen analysis, the acetolysis technique was used, modified for geopropolis. 75 pollen types were found, out of which 59 types were identified as belonging to 28 botanical families. The family Fabaceae was notable with 12 identified pollen types. The genera $\mathrm{Ce}$ cropia (Urticaceae), Eucalyptus (Myrtaceae), Mimosa pudica (Fabaceae) and Myrcia I (Myrtaceae) were present in all analyzed samples. The pollen types Protium heptaphyllum (Buseraceae) and Scbinus terebinthifolia (Anacardiaceae) were both used as a resin source by the bees and showed frequency distributions of $56.25 \%$ and $81.25 \%$, respectively.
\end{abstract}

\section{Introdução}

As abelhas-sem-ferrão (meliponíneos) possuem uma distribuição pantropical, sendo encontradas em florestas tropicais e subtropicais em todo o mundo, principalmente nos países da América do Sul (Barth, 2006; Sawaya et al., 2009; Souza et al., 2011). No Brasil, existem c. de 192 espécies de abelhas-sem-ferrão, dentre as quais se destacam as do gênero Melipona (tribo Meliponini) (Alves et al., 2006; Dutra, 2006). A espécie Melipona scutellaris L. (uruçu) é endêmica da região nordestina brasileira, ocorrendo na área compreendida entre a Bahia e o 
Rio Grande do Norte, principalmente nas zonas de transições entre a Mata Atlântica e a Caatinga (Vianna et al., 2013).

Essas abelhas são responsáveis pela polinização de diversas espécies de plantas, tendo assim fundamental importância para a biodiversidade desses biomas (Sawaya et al., 2009; Sanches, 2012). Além disso, os produtos oriundos da criação racional desses meliponíneos (mel, pólen e geoprópolis) são importantes fontes de renda na agricultura familiar (Alves et al., 2006; Vianna et al., 2013).

A geoprópolis é produzida pelas abelhas-sem-ferrão que coletam resinas vegetais, óleos essenciais, pólen, cera, terra e/ou barro usando o produto originado para vedar a colmeia, auxiliando na proteção e na manutenção da temperatura interna (Souza et al., 2011; Silva et al., 2013). Estudos que abordem a "geoprópolis" dos meliponíneos ainda são minoria, perdendo espaço para a própolis produzida pela Apis mellifera L. Contudo, houve crescimento nos últimos anos, com alguns bons resultados, principalmente em relação à intensa propriedade terapêutica da geoprópolis (Souza et al., 2011; Sanches, 2012).

O pólen está presente na geoprópolis por conta de uma contaminação, sendo trazido pelo vento ou pela própria abelha quando o transporta para o interior da colmeia (Barth e Luz 2003). Por isso, pode ser indicativo das espécies vegetais visitadas pelas abelhas para a coleta da resina vegetal, principal matéria-prima presente na composição do produto. O espectro polínico presente na geoprópolis é bastante amplo (Barros et al., 2013). Através de análise polínica, é possível identificar a origem fitogeográfica do produto, além da identificação das principais espécies vegetais que estão contribuindo para a sua composição. Essa informação é de crucial importância para que os meliponicultores tracem planos de manejo que visem ao aumento e à melhoria da produção (Sawaya et al., 2009).

No Brasil, em geral, e na Bahia, em particular, existe pouca informação sobre as plantas de importância para a produção da geoprópolis. Frente a isso, esse trabalho teve como objetivo analisar palinologicamente amostras da geoprópolis produzida por Melipona scutellaris L. em uma área da Mata Atlântica nordestina brasileira, com o intuito de fornecer dados que contribuam para o aumento do conhecimento da flora de interesse para essas abelhas na região e, assim, possibilitar aos meliponicultores informações que possam contribuir para a produção de safras mais rentáveis economicamente.

\section{Material e Métodos}

\section{Área de estudo}

As amostras foram obtidas do meliponário da Fazenda São Lucas (1201'786"S e 3804'366"W) localizado em uma região com predominância de Mata Atlântica secundária, no município de Entre Rios (Figura 1). Esse município está localizado na BR-110, entre Salvador e Alagoinhas, no estado da Bahia, nordeste do Brasil. O estado da Bahia possuiu cerca de 565 mil Km2, com 417 municípios, ocupando aproximadamente $36,4 \%$ da região nordeste (IBGE, 2002).

\section{Amostras}

Duas colônias de M. scutellaris (A e B) foram selecionadas e um total de 16 amostras coletadas, sendo oito pertencentes à colônia $\mathrm{A}$

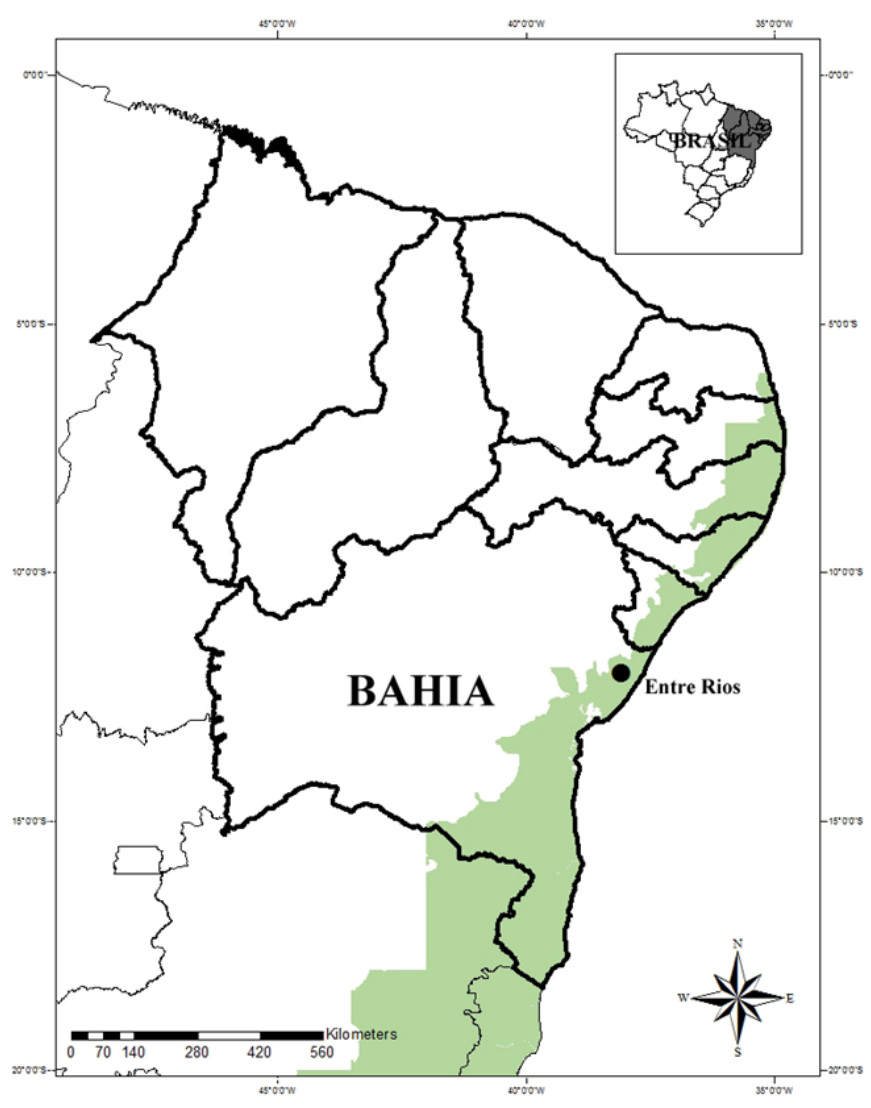

Figura 1. Mapa do Brasil com destaque para a região Nordeste, no canto superior à direita, e abaixo, destaque para o estado da Bahia, o município de Entre Rios e a distribuição do bioma Mata Atlântica (em verde).

e oito amostras à colônia B. As coletas das amostras ocorreram no período de 24 meses (julho/2012 a junho/2014) com um intervalo de três meses entre as coletas, tempo necessário para que as abelhas pudessem produzir uma nova camada de geoprópolis. Essas amostras foram coletadas através do método de raspagem total de uma área na parte externa da colmeia, tomando o cuidado de sempre coletar da mesma área.

No mês de julho de 2013, não foi possível realizar a coleta devido ao tempo chuvoso na região. Sendo assim, a referida coleta ocorreu no mês seguinte. Entretanto, conservou-se a continuidade do calendário com a próxima coleta prevista para outubro de 2013.

As amostras coletadas foram acondicionadas em recipientes hermeticamente fechados e etiquetados, sendo encaminhadas para análise no Laboratório de Micromorfologia Vegetal (LAMIV) da Universidade Estadual de Feira de Santana (UEFS).

\section{Análises palinológicas}

As amostras foram processadas de acordo com a metodologia descrita por Matos et al. (2014). Para o censo palinológico, estabeleceu-se o mínimo de 500 grãos de pólen por amostra de geoprópolis; a identificação da afinidade botânica dos tipos polínicos foi realizada de acordo com as indicações de Santos (2011).

Os grãos de pólen tiveram sua afinidade botânica determinada com o auxílio das lâminas depositadas na palinoteca do LAMIV, na qual também foram depositadas todas as lâminas preparadas. Foram também utilizados catálogos e outros trabalhos para auxiliar na 
identificação da origem botânica dos tipos polínicos. Após o término das análises, os principais tipos polínicos foram fotomicrografados em microscópio óptico.

Foi igualmente estabelecida a frequência de distribuição dos tipos polínicos, conforme Jones e Bryant (1996), categorizadas nas seguintes classes de frequência: muito frequente $>50 \%$, frequente $20-50 \%$, pouco frequente $10-20 \%$, e raro $<10 \%$.

\section{Coleta dos Dados Macroclimáticos}

Os dados macroclimáticos de temperatura e pluviosidade, referentes a todo o período de estudo, foram obtidos na estação meteorológica convencional de Alagoinhas (Bahia), através do Instituto Nacional de Meteorologia (INMET, 2014).

\section{Hábito e Recurso Floral}

Para inferência do hábito relacionado às espécies afins aos tipos polínicos identificados, seguiram-se as recomendações de Marchant et al. (2002), Modro et al. (2011) e Alves e Santos (2014). Em relação ao recurso floral disponibilizado às abelhas, baseou-se nos estudos de Dórea (2007) e Luz et al. (2007).

\section{Análise Estatística}

A análise de similaridade das amostras foi realizada através do programa estatístico PRIMER-E-Plymouth Routines In Multivariate Ecological Research, Version 6.1.6 (Clarke e Gorley 2006). O coeficiente de similaridade utilizado foi o Jaccard, por não considerar as ausências compartilhadas como evidência de similaridade e considerando os dados sobre a presença ou ausência dos tipos polínicos identificados.

Foi também utilizado o programa STATISTICA - Data Analysis Software System Version 10 (StatSoft 2011) para correlacionar os dados de precipitação pluviométrica com a quantidade de tipos polínicos identificados nos meses amostrados.

\section{Resultados}

Foram encontrados 75 tipos polínicos nas amostras analisadas de geoprópolis. Desse total, 59 tipos foram devidamente identificados e pertencem a 28 famílias botânicas (Tabela 1). Dentre elas, a família Fabaceae destacou-se com 12 tipos polínicos, seguida pela família Myrtaceae, com sete tipos. A família Rubiaceae também teve uma grande representatividade com a presença de cinco tipos no total. O número de tipos polínicos obtidos por amostra variou de 13 na amostra B-04/14 a 36 na amostra A-04/13 (Tabela 1).

Os tipos Cecropia (Urticaceae), Eucalyptus (Myrtaceae), Mimosa pudica (Fabaceae) e Myrcia I (Myrtaceae) estiveram presentes em todas as amostras analisadas. Os tipos Protium heptaphyllum (Buseraceae) e Schinus terebinthifolia (Anacardiaceae), ambos relacionados a plantas resiníferas, apresentaram, respectivamente, 56,25\% e $81,25 \%$ de frequência de distribuição no total amostral. Além desses citados, outros noves tipos (Borreria verticillata, Miconia, Mimosa tenuiflora, Myrcia II, Poaceae, Rhynchospora cepholotes, Senna, Serjania e Solanum paniculatum) foram classificados como muito frequente, pois apresentaram frequência de distribuição superior a $50 \%$ (Figuras 2 e 3).

A maior frequência de ocorrência foi do tipo Eucalyptus $(74,0 \%)$ na amostra A (08/13). Esse tipo também apresentou alta frequência em outras amostras analisadas: 61,6\% em A-04/14, $56,8 \%$ em B-04/13, 51,2\% em A-01/14 e 50,6\% em B-10/13. Os tipos Miconia (Melastomataceae) e Mimosa pudica também apresentou alta frequência, respectivamente $67,2 \%$, na amostra A-10/12 e $52,8 \%$ na amostra B-01/13 (Tabela 1$)$.

Nas amostras foram identificados os tipos Angelonia, Byrsonima sericea, Ficus hirsuta e Stigmaphyllon blanchetii, consideradas espécies fornecedoras de óleos, e os tipos Cecropia, Maytenus, Phyllanthus, Protium heptaphyllum, Schinus terebinthifolia e Spondias tuberosa como resinosas (Tabela 1).

Com relação ao hábito de crescimento das espécies botânicas correspondentes aos tipos identificados nas amostras, o mais frequente foi o arbóreo (36 táxons), seguido de arbustivo (18), herbáceo (15) e liana (quatro). As trepadeiras (dois táxons) foram as de menor representatividade nas amostras polínicas (Tabela 1).

A média da temperatura na região durante os meses de coleta variou de $23^{\circ} \mathrm{C}$ a $28^{\circ} \mathrm{C}$, com o maior registro no mês de janeiro de $2014\left(33^{\circ} \mathrm{C}\right)$ e o menor em setembro de $2012\left(18,4^{\circ} \mathrm{C}\right)$. Por sua vez, o maior volume de índice pluviométrico registrado foi de 190 $\mathrm{mm}^{3}$, em abril de 2013, com ocorrência de 26 tipos polínicos. No ano seguinte, em abril de 2014, houve diminuição na quantidade de chuvas $\left(74,4 \mathrm{~mm}^{3}\right)$ e também um aumento no número de tipos encontrados (33 tipos). O mês de janeiro de 2014 apresentou o menor índice pluviométrico $\left(21 \mathrm{~mm}^{3}\right)$, contudo foram identificados 38 tipos polínicos. Outro mês no qual houve baixa incidência de chuvas $\left(31,9 \mathrm{~mm}^{3}\right)$ foi outubro de 2012 e esse mês também apresentou uma alta quantidade de tipos polínicos (32) (Figura 4).

Não obstante, foi verificado que não houve uma correlação significativa entre a precipitação pluviométrica e a quantidade de tipos polínicos identificados em cada mês $(\mathrm{F}=0,14, \mathrm{p}<0,7, \mathrm{r} 2=$ $0,17, b=0,70)$. Assim, outros fatores podem estar influenciando na variação quantitativa de tipos polínicos na região em estudo.

As amostras B-10/13 e B-01/14 apresentaram a maior similaridade palinoflorística encontrada entre as amostras, com índice de 68,75\% (Figura 5). Elas apresentaram 21 tipos polínicos em comum. O mesmo ocorreu com as amostras A-01/13 e A-01/14, colhidas no mesmo mês em anos distintos e que apresentaram uma similaridade de c. de 61\% (Figura 5), com 16 tipos polínicos em comum. Por sua vez, as amostras B-07/12 e A-10/12 tiveram uma similaridade de 56\% (Figura 5).

\section{Discussão}

A ocorrência de 75 tipos polínicos nas amostras analisadas de geoprópolis evidencia a diversidade florística do bioma Mata Atlântica no município de Entre Rios, no qual há uma grande opção de espécies de plantas para o pasto das abelhas Melipona spp. na coleta de néctar e pólen. No estudo de Barros et al. (2013), em uma área de restinga do estado do Maranhão, os 


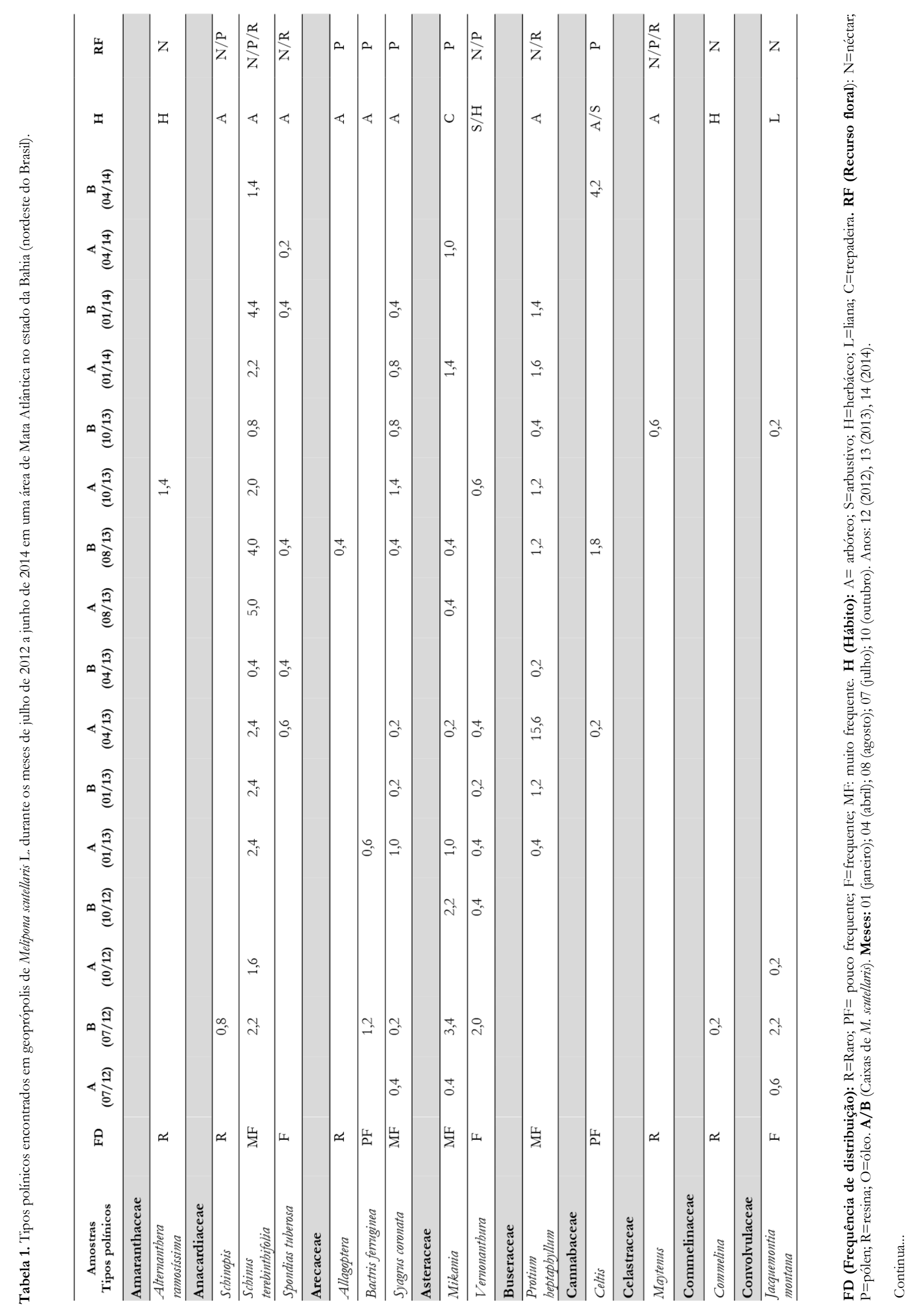




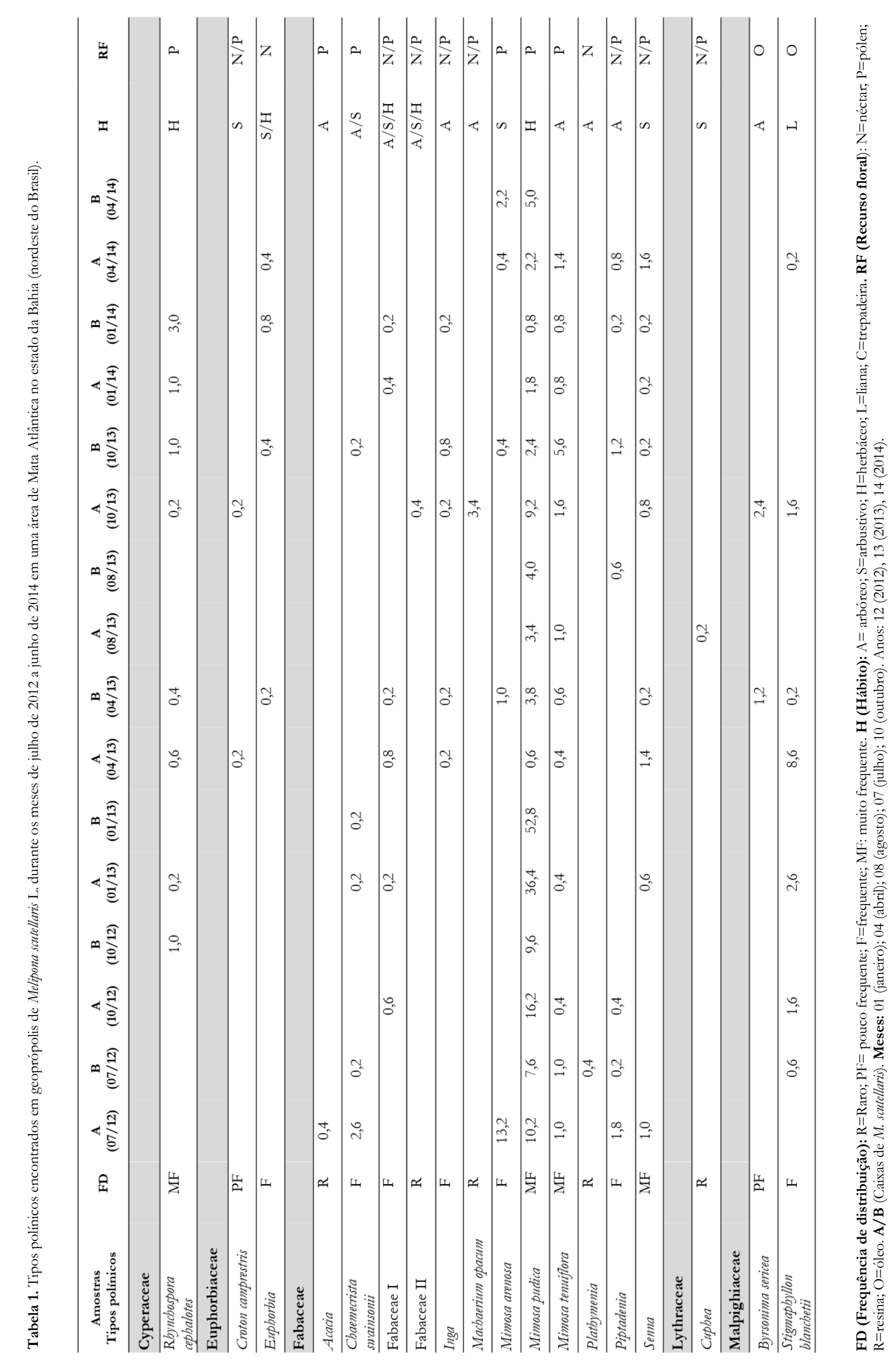




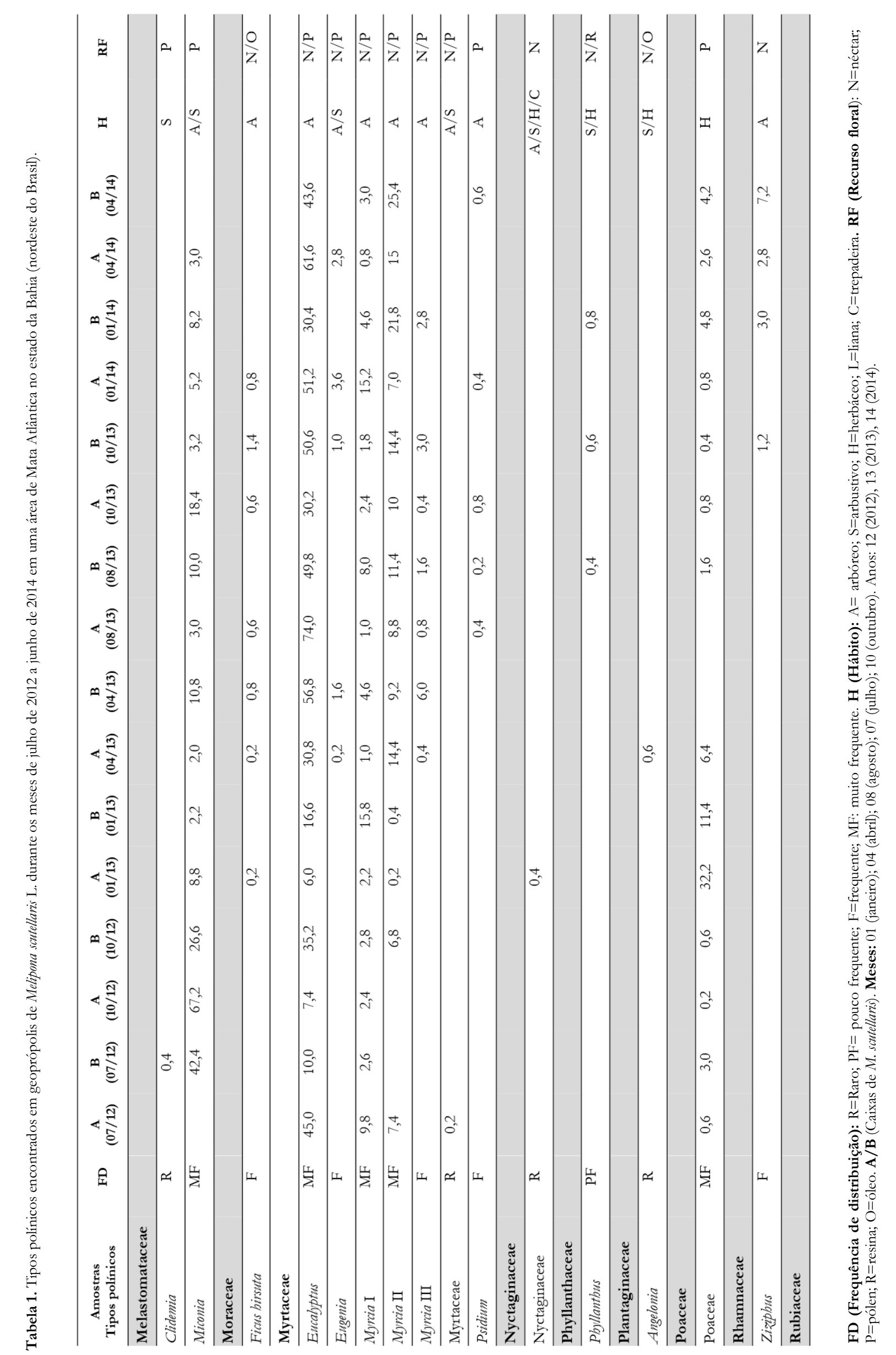




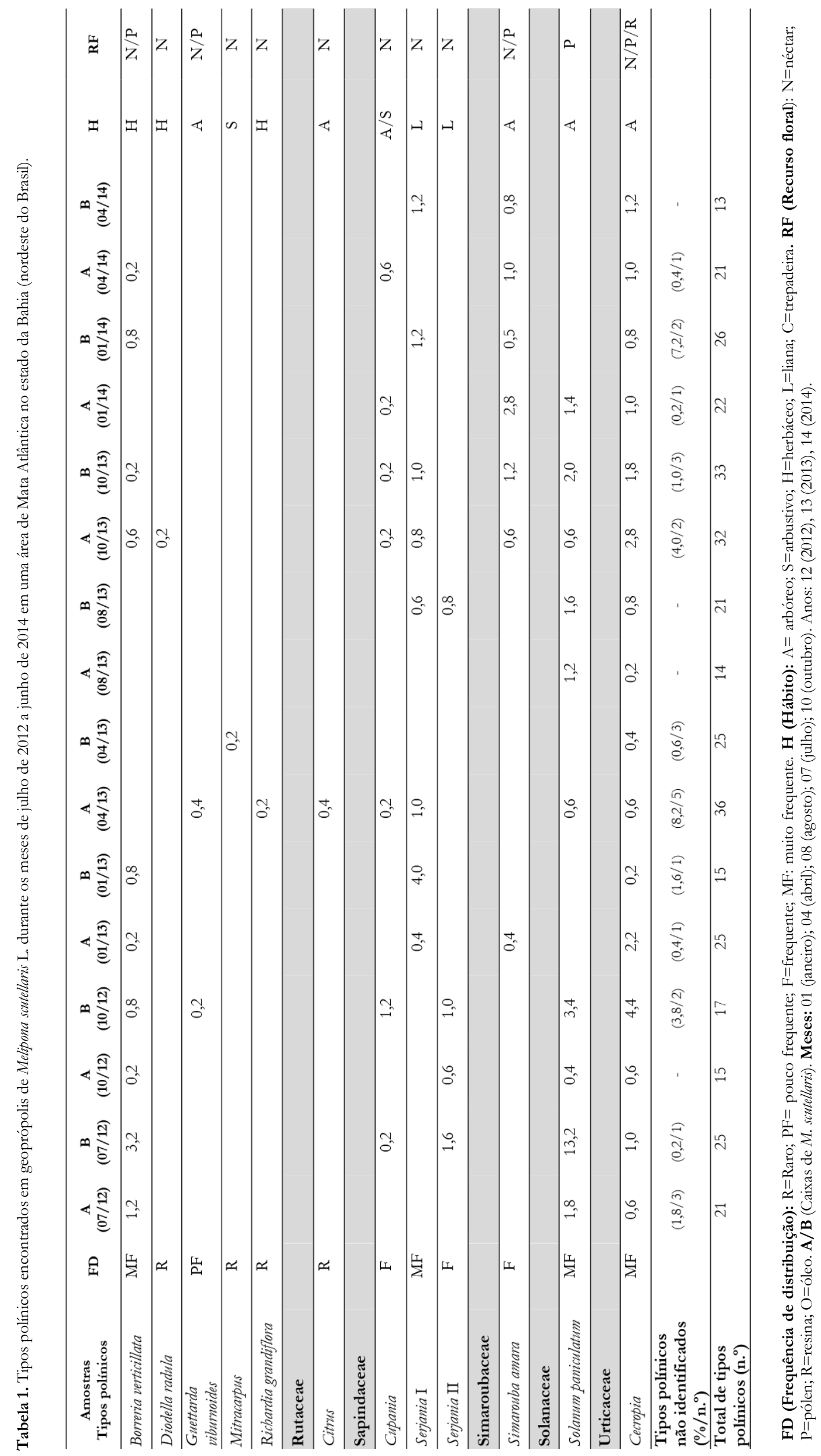




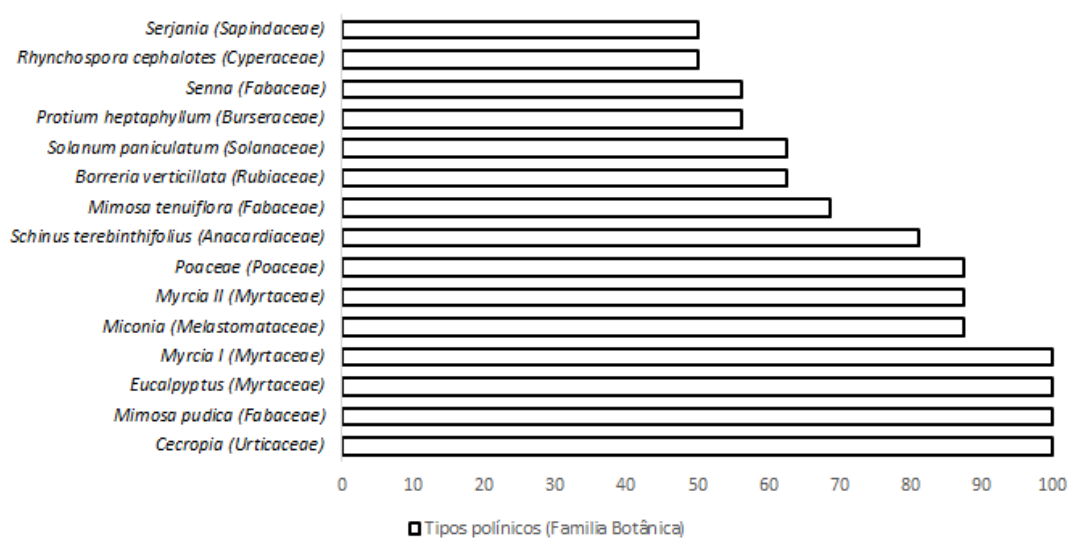

Figura 2. Tipos polínicos presentes em mais de 50\% das amostras de geoprópolis coletadas em uma área de Mata Atlântica no estado da Bahia (nordeste do Brasil).

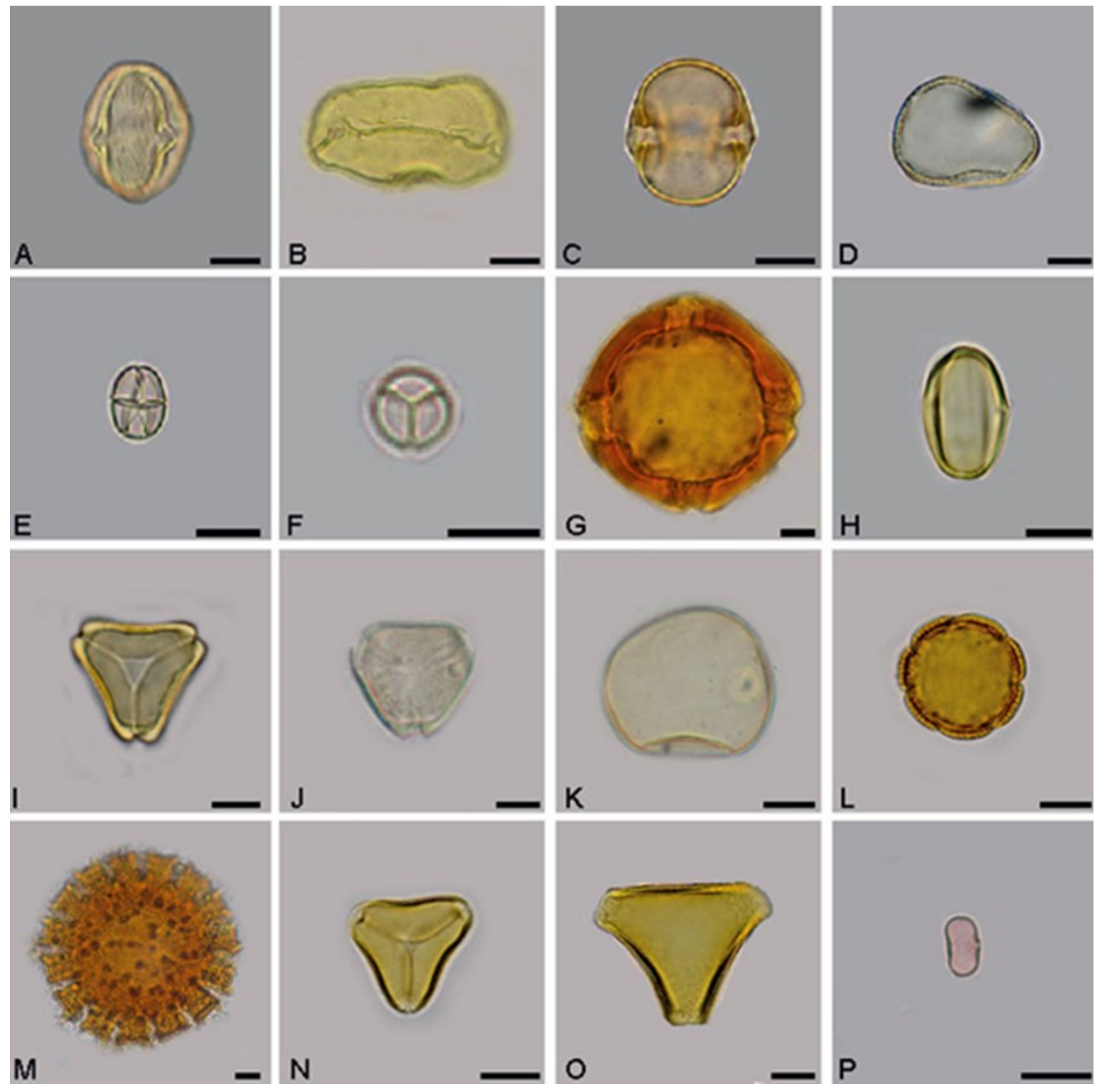

Figura 3. Tipos polínicos encontrados em amostras da geoprópolis coletadas em uma área de Mata Atlântica no estado da Bahia (nordeste do Brasil) A. Anacardiaceae: Schinus terebinthifolia. B. Arecaceae: Syagrus coronata. C. Burseraceae: Protium heptaphyllhm. D. Cyperaceae: Rhynchospora cephalotes. E-F. Fabaceae: E. Mimosa tenuiflora. F. Mimosa pudica. G. Malpighiaceae: Stigmaphyllon blanchetii. H. Melastomataceae: Miconia. I-J. Myrtaceae: I. Eucalptus. J. Myrcia. I. K. Poaceae. L-M. Rubiaceae: L. Borreria verticillata. M. Richardia grandiflora. N-O. Sapindaceae: N. Cupania. O. Serjania I. P. Urticaceae: Cecropia. (Barra $=10 \mu \mathrm{m})$ 


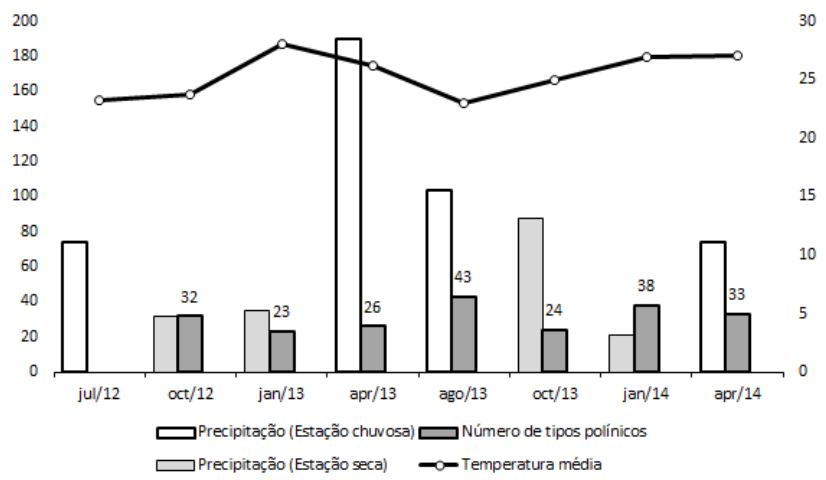

Figura 4. Relação entre a precipitação - estação seca e chuvosa -, a temperatura média e o número de tipos polínicos identificados nas amostras de geoprópolis coletadas em uma área de Mata Atlântica, no estado da Bahia (nordeste do Brasil).

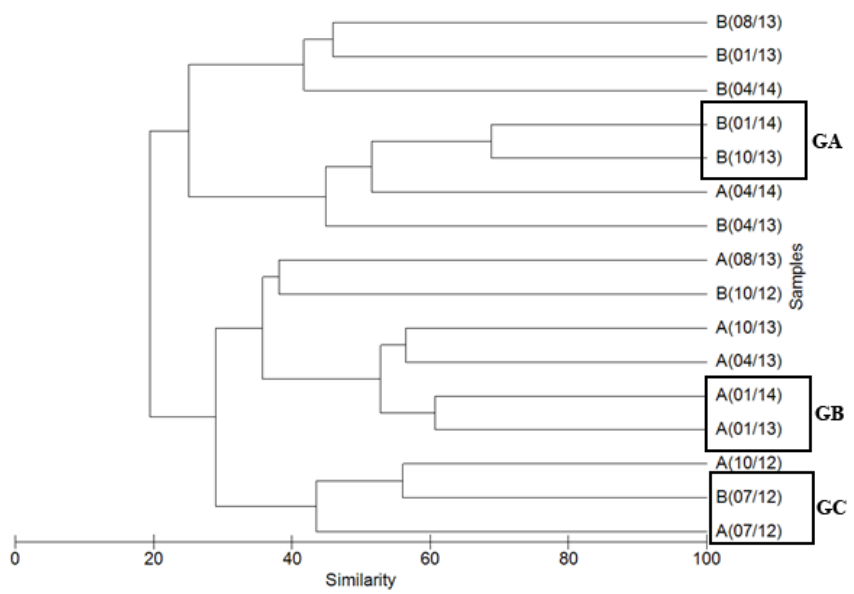

Figura 5. Dendograma de similaridade - coeficiente de Jaccard - entre as amostras de geoprópolis coletadas em área de Mata Atlântica no estado da Bahia (nordeste do Brasil).

autores encontraram 38 tipos polínicos na geoprópolis estudada, número inferior ao aqui apresentado.

Barth e Luz (2003) classificaram o tipo Eucalyptus como pólen dominante, ou seja, ele esteve presente em mais de $45 \%$ das amostras em estudos com a geoprópolis produzida no sudeste brasileiro. As espécies vegetais atribuídas a esse tipo polínico ocorrem também com bastante frequência nos estudos envolvendo a própolis e os demais produtos apícolas/meliponícolas produzidos no Brasil (Matos et al., 2014; Matos e Santos 2017a; Matos e Santos 2017b). Apesar de não ser uma espécie nativa da flora brasileira, essa espécie adaptou-se muito bem ao clima e ao solo e, desde a década de 1990, vem sendo empregada de forma extrativista em diversas regiões do país (Araújo et al., 2004).

Outro tipo polínico com uma alta distribuição nesse estudo foi o tipo Miconia, da família Melastomataceae. Tipos englobados nessa família são bastante representativos nos estudos palinológico dos produtos apícolas e, talvez, uma das explicações dessa grande representatividade deve-se à polinização dessa família ser realizada basicamente pelas abelhas, que coletam mais especificamente o pólen ofertado; contudo, algumas espécies também disponibilizam néctar como recurso (Maia, 2013).

A família Fabaceae também foi bastante representativa nesse estudo. Várias espécies vegetais dessa família desempenham um im- portante papel como elemento florístico nas mais diversas formações vegetais, principalmente nas áreas de abrangência do domínio atlântico onde ela apresenta uma ampla riqueza e abundância (Brito et al., 2010).

Os óleos não voláteis são utilizados por algumas espécies de abelhas na construção de ninhos, pois fornecem impermeabilidade à água e proteção antimicrobiana (Rosa, 2009). A abelha uruçu utiliza esse recurso como matéria-prima para a produção da geoprópolis, que reveste o exterior da colmeia (Sanches, 2012); isso foi refletido nas amostras analisadas pela presença dos tipos polínicos relacionados às Malpighiaceae (Byrsonima sericea e Stigmaphyllon blanchetii) que são grandes produtoras de óleos essenciais (Buchamann, 1987). Ressalta-se que o tipo polínico S. blanchetii, por ter sido observado ao longo dos dois anos de análises, pode então ser indicativo da principal fonte de óleo para a geoprópolis de M. scutellaris na área em estudo.

Dentre os compostos constituintes da geoprópolis, destaca-se a resina, no entanto, os fatores que possam influenciar a preferência das abelhas por determinada fonte desse recurso ainda não são conhecidos, mas elas apresentam seletividade nessa escolha (Barros et al., 2013). A depender da região de coleta, espécies de abelhassem-ferrão recolhem resinas de diferentes plantas e a presença do pólen dessas espécies na geoprópolis pode ser um excelente indicador de possíveis fontes dessa matéria-prima (Sawaya et al., 2009). As espécies Schinus terebinthifolia Raddi (Anacardiaceae) e Cecropia spp. (Urticaceae), de acordo com Matos et al. (2014), são possíveis fornecedores de resina para a própolis de Apis mellifera na região do Litoral Norte baiano, onde também está localizada a área em estudo. Pólen referente às duas espécies foi muito frequente no espectro polínico analisado, tendo o tipo Cecropia ocorrido em 100\% das amostras, enquanto que o tipo S. terebinthifolia ocorreu em 81,25\%, ambos com baixa frequência de ocorrência. Dessa forma, sinalizase que esses dois tipos podem também ser indícios de fornecedores da resina usada pela $M$. scutellaris para a produção da geoprópolis nessa região. Matos e Santos (2017a) analisaram a própolis produzida nessa mesma região e encontraram também os tipos correspondentes a essas espécies como fornecedores da resina utilizada pela A. mellifera na área.

A maior porcentagem dos tipos polínicos foi relacionada às espécies de porte arbóreo (18), seguido do herbáceo (15). O estrato arbóreo é o principal fornecedor de recurso para as mais diversas espécies de abelhas, segundo Pereira et al. (2004). Já Silva et al. (2012) observaram que a maioria das espécies englobadas nesse estrato são polinizadas pelas abelhas. Entretanto, segundo esses autores, mesmo se o estrato herbáceo apresenta menor diversidade de espécies, quando comparado com o arbóreo, quase a totalidade delas são dependentes dessa relação ecológica, demonstrando, dessa forma, a extrema importância dessas espécies para a manutenção das comunidades de abelhas.

Estatisticamente, a variação na quantidade de chuvas ocorridas na região não influenciou diretamente na quantidade de tipos polínicos encontrados nas amostras analisadas, assim como ocorreu nos estudos realizados nessa mesma área por Matos e Santos (2017ab). E isso apesar da disponibilidade de água que impulsionou a floração de algumas espécies nos meses mais chuvosos (cumpre ressaltar que a resposta da floração em um mês chuvoso é visualiza- 
da no mês seguinte). Nesses meses chuvosos, o número de tipos identificados nas amostras variou bastante, sendo que quatro deles (Acacia, Allagoptera, Celtis e Clidemia) são altamente poliníferos e o número reduzido de tipos polínicos encontrados em alguns meses pode ser decorrência do aparecimento deles. Já nos meses mais secos, seis tipos polínicos foram específicos: Alternanthera ramosissima, Maytenus, Fabaceae II, Machaerium opacum, Nyctaginaceae e Diodella radula. $\mathrm{O}$ aparecimento desses tipos nesses períodos específicos pode não ser decorrente apenas da disponibilidade da água, já que outros fatores intrínsecos à abelha ou à própria planta podem ser responsáveis por essa variação dos tipos polínicos no decorrer das estações seca e chuvosa.

De acordo com o índice de similaridade utilizado, houve semelhança entre amostras coletadas em anos distintos, assim como ocorreu com amostras obtidas no mesmo ano. Esse fato pode ser decorrente da proximidade das colônias analisadas que dispunham do mesmo "menu" florístico para forrageio. Nele, algumas "espécies-chave" (Cecropia, Mimosa pudica, Eucalyptus, Myrcia I e II, Schinus terebinthifolia, Mimosa tenuiflora, Borreria verticillata, Protium beptaphyllum, Serjania) estão presentes e a competição intercolônia é intensificada pela proximidade das mesmas. Por conta do tipo de análise utilizada, a qual não considera as ausências compartilhadas como evidência de similaridade, essas espécies podem ser as responsáveis pelos agrupamentos formados. .

\section{Conclusão}

O diagnóstico palinológico da geoprópolis, produzida pela $M$. scutellaris no município de Entre Rios (BA) no nordeste brasileiro, apresentou uma assembleia polínica bastante diversificada, destacando-se quando comparada a estudos realizados com outras espécies de abelhas-sem-ferrão. Essa assembleia é caracterizada especialmente por grãos de pólen de Schinus terebinthifolia, Protium heptaphyllum, Cecropia (resiníferas), Serjania (nectarífera), Mimosa pudica, Miconia, Poaceae, Rhynchospora cephalotes, Solanum paniculatum (poliníferas), Borreria verticillata, Eucalyptus, Myrcia I e II e Senna (nectaríferas/poliníferas).

Esse levantamento palinoflorístico indica que, na área em estudo, mesmo apresentando uma Mata Atlântica secundária em uma região onde há extensa plantação de Eucalyptus, as abelhas ainda conseguem encontrar um pasto com uma ampla variedade de espécies vegetais que fornecem os recursos necessários para a manutenção das suas atividades. Além disso, as espécies introduzidas, como Eucalyptus spp., disponibilizam também às abelhas uruçu recursos florais, inferidos pela alta frequência de ocorrência e distribuição dos respectivos tipos polínicos nas amostras estudadas.

A variedade de tipos fornecedores de óleos essenciais e resina, matéria-prima importante para a produção da geoprópolis, reforça a importância da diversidade florística da região, disponibilizando à Melipona scutellaris recursos ao longo dos períodos secos e chuvosos. O clima na região e a disponibilidade de chuvas ao longo do ano são fatores importantes; entretanto, eles não influenciaram diretamente na quantidade de tipos polínicos ao longo do período em análise, já que outros fatores, como biologia da abelha e das espécies botânicas, também influenciam diretamente no resultado final.

Apesar do meliponário em estudo estar localizado em uma área cercada pela implantação de uma agricultura extrativista, a manutenção da vegetação garantiu a disponibilidade de recurso ao longo de todo o ano. As espécies aqui listadas como fonte para $M$. scutellaris na região são de extrema importância para a continuidade de uma produção consciente da geoprópolis na área estudada. Sendo assim, é necessário conservá-las para que ocorra a manutenção e a proteção química e física da colônia.

\section{Agradecimentos}

Ao Laboratório de Micromorfologia Vegetal da Universidade Estadual de Feira de Santana pelo uso das suas instalações, à Coordenação de Aperfeiçoamento de Pessoal de Nível Superior (CAPES) - FinanceCode001 pela bolsa de doutorado concedida a VRM, e ao Conselho Nacional de Desenvolvimento Científico e Tecnológico (CNPq) pelo apoio a FARS (\# 303862 / 2013-0).

\section{Referências}

Alves RF, Santos FAR. Plant sources for bee pollen load production in Sergipe, northeast Brazil. Palynology 2014;38:90-100.

Alves RMO, Justina GD, Souza BA, Dias CS, Sodré, GS. Criação de abelhas nativas sem ferrão (Hymenoptera: Apidae): sustentabilidade na comunidade de Joia do Rio, munícipio de Camaçari, estado da Bahia. Magistra 2006;18(4):221-228.

Araújo CVM, Alves LJ, Santos OM, Alves, JM. Micorriza arbuscular em plantação de Eucalyptus cloęiana $\mathrm{F}$. Muell no litoral norte da Bahia, Brasil. Acta Botanica Brasilica 2004;18(3):512-520.

Barth OM. Palynological analysis of geoprópolis samples obtained from six species of Meliponinae in the campus of the Universidade de Ribeiro Preto, USP, Brazil. Apiacta 2006;41:71-85.

Barth OM, Luz CFP. Palynological analysis of Brazilian geopropolis sediments. Grana 2003;42(2):121-127.

Barros MHMR, Luz CFP, Albuquerque PMC. Pollen analysis of geopropolis of Melipona (Melikerria) fasciculata Smith, 1854 (Meliponini, Apidae, Hymenoptera) in areas of Restinga, Cerrado and flooded fields in the state of Maranhão, Brazil. Grana 2013;52(2):81-92.

Buchmann SL. The ecology of oil flowers and their bees. Annual Review of Ecology and Systematics 1987;18:343-369.

Brito VLG, Pinheiro M, Sazima M. Sophora tomentosa e Crotalaria vitellina (Fabaceae): biologia reprodutiva e interações com abelhas na restinga de Ubatuba, São Paulo. Biota Neotropica 2010;10 (1):185-192.

Clarke KR, Gorley RN.V6 PRIMER: manual do usuário/tutorial. PRIMER-E, Plymouth 2006;5:01-91.

Dórea M.C. O pólen armazenado por abelhas solitárias (Apidae, Centridini): Estudo em uma área de caatinga na Bahia Dissertação [Mestrado em Botânica] - Universidade Estadual de Feira de Santana; 2007.

Dutra R.P. Características físico-químicas do geoprópolis de Melipona fasciculata Smith (tiúba) produzido no estado do Maranhão. Dissertação [Mestrado em Ciências Agrárias] - Universidade Federal do Maranhão; 2006.

Instituto Brasileiro de Geografia e Estatística [nternet]. Brasília: Série Cidades 2002. [acesso em 25 set 2015] Disponível em: http://www.ibge.gov.br/cidadesat/default.php/

Instituto Nacional de Meteorologia [Internet]. Estações convencionais 2014. [acesso em 25 set 2015] Disponível em: http:// www.inmet.gov.br

Jones GD, Bryant VM. Melissopalynology. In: Jansonius J, McGregor DC (eds.). Palynology: principles and applications. St. Louis: AASP Foundation; 1996. p. 933-938 
Luz CFP, Thomé ML, Barth OM. Recursos tróficos de Apis mellifera L. (Hymenoptera, Apidae) na região de Morro Azul do Tinguá, estado do Rio de Janeiro. Revista Brasileira de Botânica 2007;30:29-36.

Marchant R, Almeida L, Behling H, Berrio JC, Bush M, Cleef A, Duivenvoorden J, Kappelle M, Oliveira P, Oliveira-Filho AT, Lozano-Garcia S, Hooghiemstra H; Ledru MP, LudlowWiechers B, Markgraf V, Mancini V, Paez M, Prieto A, RangeL $\mathrm{O}$, Salgado-Labouriau ML.Distribution and ecology of parent taxa of pollen lodged within the Latin American Pollen Database. Review of Palaeobotany and Palynology 2002;121:1-75.

Maia FR. Sistemas reprodutivos e visitantes florais em Melastomataceae dos campos rupestres no limite sul do cerrado, Tibagi, Paraná. Dissertação [Mestrado em Botânica] -Universidade Federal do Paraná; 2013.

Matos VR, Santos FAR. The pollen spectrum of the propolis of Apis mellifera L. (Apidae) from the Atlantic Rainforest of Bahia, Brazil. Palynology 2017a;41(2):207-215.

Matos VR, Santos FAR. Pollen in honey of Melipona scutellaris L. (Hymenoptera: Apidae) in an Atlantic rainforest area in Bahia, Brazil. Palynology 2017b;41(1):144-156.

Matos VR, Alencar SM, Santos FAR. Pollen types and levels of total phenolic compounds in própolis produced by Apis mellifera L. (Apidae) in an área of the semiarid region of Bahia, Brazil. Anais da Academia Brasileira de Ciências 2014;86(1): 407-418.

Modro AFH, Message D, Luz CFP, Neto JAAM. Flora de importância poliníferas para Apis mellifera (L.) na região de Viçosa, MG Revista Árvore 2011;35:1145-1153.

Pereira FM, Freitas BM, Alves JE, Camargo RCR, Lopes MTR, Vieira-Neto JM, Rocha RS. Flora apícola do Nordeste; 2004.

Rosa JF. Dinâmica espacial na diversidade de abelhas Centridini: oferta de óleos florais como medida da qualidade do habitat. Dissertação [Mestrado em Ecologia e Biomonitoramento] Universidade Federal da Bahia; 2009.

Sanches MA. A própolis de abelhas-sem-ferrão e suas propriedades terapêuticas. Pesquisa e Tecnologia 2012; 9(1):1-6.

Santos FAR. Identificação botânica do pólen apícola. Magistra 2011;23:4-9.

Sawaya ACHF, Calado JCP, Santos LC, Marcucci MC, Akatsu IP, Soares AEE, Abdelnur PV, Cunha IBS, Eberlin MN. Composition and antioxidant activity of própolis from three species of Scaptotrigona stingless bees. Journal of ApiProduct and ApiMedical Science 2009;1(2):37-42.

Silva ECC, Muniz MP, Nunomura RCS. Constituintes fenólicos e atividade antioxidante da geoprópolis de duas espécies de abelhas -sem-ferrão amazônicas. Química Nova 2013;36(5):628-633.

Silva CI, Araújo, G, Oliveira, PEAM. Distribuição vertical dos sistemas de polinização bióticos em áreas de cerrado sentido restrito no Triângulo Mineiro, MG, Brasil. Acta Botanica Brasilica 2012;26(4):748-760.

Souza LS, Alves RMO, Carvalho CAL, Souza LS, Lima-Junior CA. Produção de geoprópolis sob diferentes métodos de coletas em colônias de Melipona scutellaris Latreille (Hymenoptera: Apidae). Magistra 2011;23:10-13.

STATSOFT [Internet] STATISTICA (data analysis software system), version 10. 2011. [acesso em 30 set. 2014]. Disponível em: http://www.statsoft.com

Vianna JL, Carvalho CAL, Waldschmidt AM. Genetic variability in Melipona scutellaris from Recôncavo, Bahia, Brazil. Genetics and Molecular Research 2013;12(3): 3444-3454.
Diagnóstico polínico de la geoprópolis de Melipona scutellaris L. (Meliponini, Apidae, Hymenoptera) recogida en un área de Mata Atlántica en el nordeste de Brasil

Melipona scutellaris (urucu) es endémica de la región noreste de Brasil, poliniza un numero diverso de especies de plantas y es importante para mantener la biodiversidad de los biomas en el área, como el Bosque Atlántico. El presente trabajo analizó 16 muestras de geopropolis producidas por esta abeja en un área del Bosque Atlántico en el municipio de Entre Ríos (Babia). Para el análisis de polen, se utilizó la técnica de acetólisis, modificada para geopropolis. Se encontraron 75 tipos de polen, de los cuales 59 fueron identificados como pertenecientes a 28 familias botánicas. La familia Fabaceae fue notable con 12 tipos de polen identificados. Los géneros Cecropia (Urticaceae), Eucalyptus (Myrtaceae), Mimosa pudica (Fabaceae) y Myrcia I (Myrtaceae) estuvieron presentes en todas las muestras analizadas. Los tipos de polen $\underline{\text { Protium heptaphyllum (Buseraceae) y Schinus terebinthifolia }}$ (Anacardiaceae) se utilizaron como fuente de resina por las abejas, y tuvieron frecuencias de distribución de $56.25 \%$ y $81.25 \%$, respectivamente.

Palabras clave: Abejas nativas. Melissopalinología. Productos de las abejas. Plantas resinosas.

Diagnostic par pollen du géopolis de Melipona scutellaris L. (Meliponini, Apidae, Hymenoptera) collecté dans une zone de forêt atlantique du nord-est du Brésil

Melipona scutellaris (urucu) est endémique de la région nord-est du Brésil, pollinise un grand nombre d'espèces de plantes et joue un rôle important dans le maintien de la biodiversité des biomes de la région, tels que la forêt atlantique. Le présent travail a analysé 16 échantillons de géopropolis produits par cette abeille dans une zone de la forêt atlantique de la municipalité de Entre Rios (Babia). Pour l'analyse du pollen, une technique d'acétolyse a été utilisée, qui a été modifiée pour la géopropolis. 75 types de pollen ont été trouvés, dont 59 ont été identifiés comme appartenant à 28 familles botaniques. La famille Fabaceae était remarquable avec 12 types de pollen identifiés. Les types Cecropia (Urticaceae), Eucalyptus (Myrtaceae), Mimosa pudica (Fabaceae) et Myrcia I (Myrtaceae) étaient présents dans tous les échantillons analysés. Les types de pollen Protium beptaphyllum (Buseraceae) et Schinus terebinthifolia (Anacardiaceae), tous deux utilisés comme source de résine par les abeilles, avaient des fréquences de distribution respectives de $56,25 \%$ et $81,25 \%$.

Mots clés: Abeilles indigènes. Mélissopalinologie. Produits apicoles. Plantes résineuses. 
Este artigo possui erratum disponível em: https://doi.org/10.33447/paubrasilia.2021.e0073er

This article has erratum available at: https://doi.org/10.33447/paubrasilia.2021.e0073er

Este artículo tiene erratum publicada en: https://doi.org/10.33447/paubrasilia.2021.e0073er

\section{ERRATUM}

No artigo "Diagnóstico polínico da geoprópolis de Melipona scutellaris L. (Meliponini, Apidae, Hymenoptera) coletada em uma área de Mata Atlântica no Nordeste do Brasil”", com número de doi: 10.33447/paubrasilia.v2i1.19, publicado na revista Paubrasilia, 2(1):6-16, na página 13. Correção na legenda da Figura 3.

onde se lia:

E. Mimosa pudica. F. Mimosa tenuiflora.

leia-se:

E. Mimosa tenuiflora. F. Mimosa pudica. 